\title{
A GENERAL DECOMPOSITION THEORY FOR RANDOM CASCADES
}

\author{
EDWARD C. WAYMIRE AND STANLEY C. WILLIAMS
}

\begin{abstract}
This announcement describes a probabilistic approach to cascades which, in addition to providing an entirely probabilistic proof of the KahanePeyriere theorem for independent cascades, readily applies to general dependent cascades. Moreover, this unifies various seemingly disparate cascade decompositions, including Kahane's T-martingale decomposition and dimension disintegration.
\end{abstract}

\section{BRIEF HISTORY OF THE PROBLEM}

A theory of positive T-martingales was introduced in [K3] as the general framework for independent multiplicative cascades and random coverings. This includes spatial distributions of interest in both probability theory and in the physical sciences, e.g. [CCD, CK, DE, DG, DF, DM, F, GW, MS, MW, TLS, PW, S]. For basic definitions, let $T$ be a locally compact metric space with Borel sigmafield $\mathscr{B}$, and let $(\Omega, \mathscr{F}, P)$ be a probability space together with an increasing sequence $\mathscr{F}_{n}, n=1,2, \ldots$, of sub-sigmafields of $\mathscr{F}$. A positive $T$-martingale is a sequence $\left\{Q_{n}\right\}$ of non-negative functions on $T \times \Omega$ such that: (i) for each $t \in T,\left\{Q_{n}(t, \cdot): n=1,2, \ldots\right\}$ is a martingale adapted to $\mathscr{F}_{n}, n=1,2, \ldots$; (ii) for $P$-a.s. $\omega \in \Omega,\left\{Q_{n}(\cdot, \omega): n=1,2, \ldots\right\}$ is a sequence of Borel measurable nonnegative real-valued functions on $T$.

Let $M^{+}(T)$ denote the space of positive Borel measures on $T$, and suppose that $\left\{Q_{n}(t)\right\}$ is a positive $T$-martingale. For $\sigma \in M^{+}(T)$ let $Q_{n} \sigma$ denote the random measure defined by $Q_{n} \sigma<<\sigma$ and $\frac{d Q_{n} \sigma}{d \sigma}(t):=Q_{n}(t), t \in T$. Denote the space of bounded continuous functions on $T$ by $C_{B}(T)$. Then, essentially by the martingale convergence theorem, one obtains a random Borel measure $Q_{\infty} \sigma$ such that with probability one [K3]

$$
\lim _{n \rightarrow \infty} \int_{T} f(t) Q_{n} \sigma(d t)=\int_{T} f(t) Q_{\infty} \sigma(d t), \quad f \in C_{B}(T) .
$$

As suggested by the notation, one may view $\sigma \rightarrow \sigma_{\infty} \equiv Q_{\infty} \sigma$ as a random operator acting on $\mathrm{M}^{+}(T)$. The following special case of multiplicative cascades is central to the general theory developed in [K3].

Independent cascades. Let $b \geq 2$ be a natural number, referred to as a branching number, and let $T=\{0,1, \ldots, b-1\}^{\mathbf{N}}$ be the metric space for the ultrametric $\rho(s, t)=b^{-a(s, t)}$ where $a(s, t)=\inf \left\{n: s_{n} \neq t_{n}\right\}, s=$ $\left(s_{1}, s_{2}, \ldots\right), t=\left(t_{1}, t_{2}, \ldots\right)$. The countable set $T^{*} \equiv T^{*}(\infty):=\cup_{n=0}^{\infty} T^{*}(n)$, where $T^{*}(n):=\cup_{k=1}^{n}\{0,1, \ldots, b-1\}^{k}$, provides a convenient labelling of the

Received by the editors June 14, 1993.

1991 Mathematics Subject Classification. Primary 60G57, 60G30, 60G42; Secondary 60K35, 60D05, 60J10, 60G09.

Key words and phrases. Martingale, Hausdorff dimension, tree, random measure. 
vertices of the infinite $b$-ary tree. It is sometimes convenient to adjoin a root vertex denoted $\varnothing$ to $T^{*}$. For $t \in T, n \geq 1$, write $t \mid n:=\left(t_{1}, t_{2}, \ldots, t_{n}\right) \in$ $T^{*}, t \mid 0:=\varnothing$. For $\gamma=\left(\gamma_{1}, \ldots, \gamma_{n}\right) \in T^{*}, j \in\{0,1, \ldots, b-1\}$, define $|\gamma|:=n$ and $\gamma * j:=\left(t_{1}, \ldots, t_{n}, j\right)$. Also denote the $n$th generation partition by $\Delta_{\gamma}:=\left\{t \in T:\left.t\right|_{|\gamma|}=\gamma\right\}$. In this context the cascade generators are furnished by a (denumerable) family of i.i.d. nonnegative mean-one random variables $\left\{W_{\gamma}: \gamma \in T^{*}\right\}$ indexed by the tree and defined on a probability space $(\Omega, \mathscr{F}, P)$. With this, let

$$
P_{n}(t):=\sum_{|\gamma|=n} W_{\gamma} 1_{\Delta_{y}}(t)
$$

Now the homogeneous independent cascade is the positive $T$-martingale defined by $Q_{n}=P_{1} P_{2} \cdots P_{n}$ with respect to $\mathscr{F}_{n}:=\sigma\left\{W_{\gamma}:|\gamma| \leq n\right\}$. Define $\lambda_{\infty}:=Q_{\infty} \lambda$, where here and throughout $\lambda$ denotes the Haar measure on $T$. It may happen that $\lambda_{\infty}=0$ a.s., referred to as a degeneracy. The onset of nondegeneracy may be viewed as a critical phenomenon associated with the cascade. The fundamental theorem of [KP] on the structure of homogeneous independent cascades provides necessary and sufficient conditions on the distribution of the generators for (i) nondegeneracy and, (ii) divergence of moments of $\lambda_{\infty}[0,1]$, and provides in all cases of nondegeneracy (iii) the Hausdorff dimension of the support. Replacement of the generators at each generation by i.i.d. nonnegative random vectors $\mathbf{W}:=\left(W_{0}, \ldots, W_{b-1}\right), E \frac{1}{b} \sum W_{j}=1$, is possible without substantial changes in the conditions [N]. Moreover, this makes it possible to include the random distribution functions studied by [DF]. The results described in this note provide an entirely probabilistic approach to the computation of the finescale properties of random cascades, which also includes a simple new solution to problems (i)-(iii) for independent cascades. As will be seen, the power of this approach for more general cascades is that fine-scale computations are reduced to the law of large numbers (ergodic theory) problems.

Much of the recent focus of the study of independent cascades, both theoretically and empirically, has concerned various characteristics of their singularity structure, e.g. [HW, CK, F, PW, MS, O]. In this regard, for an arbitrary Borel measure $\sigma$, one has a unique disintegration $\sigma(\cdot)=\int \sigma_{\alpha}(\cdot) \nu(d \alpha)$ (properly interpreted), where $\int_{[0, \beta]} \sigma_{\alpha} \nu(d \alpha)$ is supported by a set of Hausdorff dimension no larger than $\beta$, and $\int_{(\beta, \infty]} \sigma_{\alpha}(B) \nu(d \alpha)>0 \Rightarrow \operatorname{dim}(B)>\beta[C, \mathrm{KK}]$. The measure $\nu$ is called the dimension spectrum. Each spectral mode $\sigma_{\alpha}$ is supported on a set of dimension at most $\alpha$. If $\nu$ has an atom at $\alpha$, then $\sigma_{\alpha}$ is unidimensional; i.e. the dimension spectrum of $\sigma_{\alpha}$ is a Dirac point mass. It follows from [KP] that the (homogeneous) independent cascades are a.s. unidimensional. The richness of extensions of the cascade theory to dependent cascades is partly reflected in an often natural nontrivial spectral disintegration.

\section{MAIN Results}

The original proof of the Kahane-Peryière theorem is based on a combination of probabilistic and analytic computations which make strong use of the statistical independence. The point of focus in [KP] is a distributional fixedpoint equation for the total mass of the cascade. Extensions to a limited class of 
dependent cascades, namely, finite-state Markov generators, have been found along similar lines in [WW1]. However, even in the countably infinite-state Markov context, the fixed-point analysis does not readily extend to dependent generators. It is interesting to note that the fixed-point equation also arises in certain interacting particle context, [HL, DL]. The approach described in this note may be of independent interest when applied to the fixed-point problem under dependence.

To define the general dependent random cascade, we begin with a probability measure $p_{0}(d x)$ and a collection of mean-one transition probability kernels $q_{n}\left(d x \mid x_{0}, \ldots, x_{n-1}\right)$, on $\mathscr{B}[0, \infty), \quad x_{i} \geq 0, n \geq 1$. Using the Kolmogorov extension theorem, one may construct a unique probability measure $P$ on the product space $\Omega:=[0, \infty)^{T^{*}(\infty)},:=:=\mathscr{B}^{T^{*}(\infty)}[0, \infty)$ together with the coordinate projection random variables $W_{\gamma}(\omega):=\omega_{\gamma}$ such that $(\mathrm{i}) W_{\varnothing}$ has distribution $p_{0}(d x)$; (ii) for any $t \in T$, the conditional distribution of $W_{t \mid n+1}$ given $W_{\gamma},|\gamma| \leq n$, is $q_{n+1}\left(d x \mid W_{t \mid 0}, W_{t \mid 1}, \ldots, W_{t \mid n}\right)$; and (iii) for $s \in T, s \mid n+1 \neq$ $t \mid n+1, W_{s \mid n+1}$ is conditionally independent of $W_{t \mid n+1}$ given $W_{\gamma},|\gamma| \leq n$ [WW2]. We refer to this model as the cascade generator corresponding to the given transition kernels $q_{n}$ and initial distribution $p_{0}$. By relabeling the states, the two-state "Markov chains on trees" constructed in [Pr, Sp] may be adapted as a special case to illustrate this general setting.

Given a cascade generator, one can define a positive $T$-martingale by applying the formula (1.2). The resulting random measure $\lambda_{\infty}:=Q_{\infty} \lambda$ will be referred to as the dependent cascade. Our approach to the study of cascades in this generality is based on three elements, namely, (i) a weight system perturbation, (ii) a size-bias transform, and (iii) a general percolation method.

Definition 2.1. A weight system is a family $\mathbf{F}$ of real-valued functions $F_{\gamma}: \Omega \rightarrow$ $[0, \infty)$ indexed by the tree, where for each $\gamma \in T^{*}, F_{\gamma}$ is $\sigma\left\{W_{\gamma \mid j}: j \leq|\gamma|\right\}$ measurable, such that $Q_{\mathbf{F}, n}(t):=\sum_{|\gamma|=n} \prod_{j \leq n}\left(W_{\gamma \mid j}\right) F_{\gamma} 1_{\Delta_{\gamma}}$ is a positive $T$-martingale, referred to as a weighted cascade. A weight system $\mathbf{F}$ is called a weight decomposition in the case $\mathbf{F}^{c}:=\left\{1-F_{\gamma}: \gamma \in T^{*}\right\}$ is also a weight system.

Note that a weight system is a weight decomposition if and only if the weights are bounded between 0 and 1 .

Assuming that one already has a nondegenerate limit cascade, Peyrière [P] defines a probability $\mathscr{Q}$ on $\Omega \times T$, named the Peyriere probability in [K1], for the joint distribution of a randomly selected path and the cascade generators along the path. This probability plays an important role in the analysis of the structure of independent cascades provided nondegeneracy has been established. The following probability is a useful extension of this notion in two directions: namely, (i) it does not require an a priori nondegeneracy condition; and (ii) it permits perturbations by a weight system. For a given weight system $\mathbf{F}$, let $Q_{\mathbf{F}, n}(t):=b^{-n} \prod_{j \leq n}\left(W_{t \mid j}\right) F_{t \mid n}, \quad t \in T, n \in \mathbf{N}$. Define a sequence $\mathscr{Q}_{\mathbf{F}, n}$ of measures on $\Omega \times T$ by

$$
\int_{\Omega \times T} f(\omega, t) \mathscr{Q}_{\mathbf{F}, n}(d \omega \times d t):=E_{P} \int_{T} f(\omega, t) Q_{\mathbf{F}, n}(t) \lambda(d t),
$$


for bounded measurable functions $f$. Then one normalizes the masses of the $\mathscr{Q}_{F, n}$ by a factor $Z_{\varnothing}:=E W_{\varnothing} F_{\varnothing}$ and extends to a probability $\mathscr{Q}_{F}$ using the Kolmogorov extension theorem. One requires $Z_{\varnothing}>0$ here and throughout.

The third ingredient to this theory is a generalization of an idea considered without proof in [K1], which may be viewed as a percolation method; see [WW2] for proofs. By independently pruning the tree, one studies the critical parameters governing the survival of mass, i.e. nondegeneracy of the percolated cascade, to determine dimension estimates on the support of the cascade. This is similar to an idea explored in [L] but differs by the distinction between locations and amounts of positive mass.

The following results provide the foundations for the general theory being announced here.

Theorem 2.1 (A Lebesgue decomposition). Let $\pi_{\Omega}$ denote the coordinate projection map of $\Omega \times T$ onto $\Omega$. Then

$$
d \mathscr{Q}_{\mathbf{F}} \circ \pi_{\Omega}^{-1}=Z_{\not}^{-1} \lambda_{\mathbf{F}, \infty}(T) 1\left(\lambda_{\mathbf{F}, \infty}(T)<\infty\right) d P+1\left(\lambda_{\mathbf{F}, \infty}(T)=\infty\right) d \mathscr{Q}_{\mathbf{F}} \circ \pi_{\Omega}^{-1}
$$

where $\lambda_{\mathbf{F}, \infty}=Q_{\mathbf{F}, \infty} \lambda$.

Theorem 2.2 (A size-biased disintegration). Given a weighting system $\mathbf{F}$,

$$
\mathscr{Q}_{\mathbf{F}}(d \omega, d t)=P_{\mathbf{F}, t}(d \omega) \lambda(d t),
$$

where

$$
\left.\frac{d P_{\mathbf{F}, t}}{d P}\right|_{\mathscr{F}_{n}}=\prod_{j \leq n}\left(W_{t \mid j}\right) F_{t \mid n}
$$

Theorem 2.3 (A submartingale bound). Let $\mathscr{F}_{t, n}:=\sigma\left\{W_{t \mid i}, W_{\gamma}: i \geq 0,|\gamma| \leq n\right\}$. Fix $c_{k} \geq 0$, such that $c_{k}$ is $\mathscr{F}_{t, 0}$-measurable, $E_{\mathscr{G}_{\mathrm{F}}} \sum_{k} c_{k}<\infty$. Given a weight system $\mathbf{F}$, letting $\lambda_{\mathbf{F}, n}=Q_{\mathbf{F}, n} \lambda$, one has for arbitrary $t \in T$

$$
b^{-n} \prod_{i \leq n}\left(W_{t \mid i}\right) F_{t \mid n} \leq \lambda_{\mathbf{F}, n}(T) \leq b^{-n} \prod_{i \leq n}\left(W_{t \mid i}\right) F_{t \mid n}+\sup _{j}\left(\frac{\prod_{i \leq j}\left(W_{t \mid i} b^{-1}\right)}{c_{j}}\right) M_{n},
$$

where

$$
M_{n}=\sum_{j=0}^{n-1} c_{j} b^{-(n-j)} \sum_{\substack{|\gamma|=n-j \\ \gamma(1) \neq(1)+1)}} \prod_{i=1}^{n-j}\left(W_{[t \mid j]] *[\gamma \mid i]}\right) F_{\gamma}
$$

is a nonnegative $P_{\mathrm{F}, t}$-submartingale with respect to $\mathscr{F}_{t, n}$, whose Doob decomposition has the predictable part $A_{n}=\frac{b-1}{b} \sum_{j \leq n-1} c_{j}$.

Theorem 2.4 (A percolation method). Let $Q_{\beta, n}(t), t \in T$, be the $\beta$-model defined by the cascade with independent generators

$$
W_{\gamma}:=B_{\gamma}= \begin{cases}b^{\beta} & \text { w. prob. } b^{-\beta}, \\ 0 & \text { else }\end{cases}
$$


and independent of the weighted cascade $Q_{\mathrm{F}, n}(t)$. Then $Q_{n}(t):=Q_{\beta, n}(t) Q_{\mathrm{F}, n}(t)$ is a weighted cascade, with weights $\mathbf{F}_{\beta}$ defined by $\left(F_{\beta}\right)_{\gamma}:=B_{\gamma} F_{\gamma}$, such that for $\sigma \in M^{+}(T)$

$$
Q_{\infty} \sigma=Q_{\beta, \infty}\left(Q_{\mathrm{F}, \infty} \sigma\right) \text { a.s. }
$$

One way in which the significance of this approach is illustrated is by the existence of the following natural weight systems.

Theorem 2.5 (Kahane decomposition). The Kahane decomposition [K2, K4], $Q_{n}(t)=Q_{n}^{\prime}(t)+Q_{n}^{\prime \prime}(t)$, is equivalent to a weight decomposition with respect to the weights defined by $F_{t \mid n}=\frac{Q_{n}^{\prime}(t)}{Q_{n}(t)}, t \in T$, and $1-F_{t \mid n}$ for $Q_{n}^{\prime}(t), Q_{n}^{\prime \prime}(t)$ respectively.

Theorem 2.6 (Dimension decomposition). The decomposition $[\mathrm{KK}, \mathrm{C}] \lambda_{\infty}=$ $\int\left(\lambda_{\infty}\right)_{\alpha} \nu(d \alpha)$ is equivalent to a weight decomposition with respect to the weight systems $\mathbf{F}_{s}:=\left\{F_{s, \gamma}: \gamma \in T^{*}\right\}, s \in[0,1]$, defined by

$$
F_{s, \gamma}=\frac{E\left[\nu_{\gamma}([0, s]) \mid \mathscr{F}_{n}\right]}{\lambda_{n}\left(\Delta_{\gamma}\right)},
$$

where

$$
\nu_{\gamma}([0, s]):=\int_{[0, s]}\left(\lambda_{\infty}\right)_{\alpha}\left(\Delta_{\gamma}\right) \nu(d \alpha), \quad n=|\gamma| .
$$

Remark 1. Two interesting classes of dependent cascades are: (i) Markov cascades and, (ii) exchangeable cascades. One may compute the weighting decompositions for Markov cascades, for example, in terms of harmonic measures corresponding to hitting probabilities of the survival classes introduced in [WW1]. Also for exchangeable cascades one may compute these weighting decompositions in terms of conditioned de Finetti measures applied to a partition of $M^{+}(T)$.

Remark 2. One may show that the maps $s \rightarrow F_{s, \gamma}$ are nondecreasing in $s$ and the Lebesgue-Stieltjes measures associated with the maps $s \rightarrow \int F_{s, t \mid n} \lambda_{n}(d t)$ converge vaguely to the spectral measure $\nu$. In fact, if the map $s \rightarrow F_{s, \gamma}$ is absolutely continuous with respect to some measure $\sigma$, then $t \rightarrow \int \frac{d F_{t, \gamma}}{d \sigma} \lambda_{n}(d \gamma)$ is itself a positive $T=[0, \infty)$-martingale. In this case the percolation method of Theorem 2.4 may be applied to compute the dimension spectrum. This, in fact, illustrates a more general notion of differentiable weights which are introduced to compute more detailed dimension spectra in [WW3].

Finally, the scope of the theory is well illustrated by the following approach to the Kahane-Peyrière theorem. In particular, the suitability of this method for more general cascades is made transparent by the suppressed role of independence beyond the martingale structure and/or the general ergodicity under the size-biased distribution.

Consider the independent cascade. Let $W$ denote a generic generator with distribution $q(d x)$. We write $\mathbf{F}=1$ to denote the case of unit weights.

A sufficient condition for nondegeneracy. Let us first show how it follows that $E_{P} W \log _{b} W<1$ is a sufficient condition for nondegeneracy. Choose $0<$ $\log _{b} c<1-E_{P} W \log _{b} W$. Fix $t \in T$. Under $P_{1, t}$, for $\gamma$ 's along the $t$-path, i.e. $\gamma=t \mid j$, some $\mathrm{j}$, the $W_{\gamma}$ 's are i.i.d. with distribution $x q(d x)$; thus the name "sized-biased". For $\gamma$ 's off of the $t$-path, i.e. $\gamma \neq t \mid j$, the $W_{\gamma}$ 's are 
i.i.d. with distribution $q(d x)$. It follows from the SLLN (i.e. ergodicity) that $P_{1, t}$-a.s., $\sqrt[i]{\frac{c^{j}}{b j} \prod_{i=1}^{j} W_{t \mid i}} \rightarrow \frac{c}{b} e^{E_{P} W \log W}$. Now use Theorem 2.3, with $c_{j}=c^{-j}$, to conclude that $\lambda_{\infty}(T)<\infty, P_{1, t}$-a.s., and therefore, by Theorem 2.2, after integrating out $\mathrm{t}, \lambda_{\infty}(T)<\infty, \mathscr{Q}_{1}$-a.s. Now apply Theorem 2.1 to see that $E \lambda_{\infty}(T)=1$.

A necessary condition for nondegeneracy. To see why the entropy condition $E_{P} W \log _{b} W<1$ is also necessary for nondegeneracy, suppose that $E_{P} W \log _{b} W$ $\geq 1, P_{t}(W=b)<1$. Fix $t \in T$. If $E_{P} W \log _{b} W>1$, then one obtains $\lambda_{n}(T) \rightarrow \infty P_{1, t}$-a.s. directly from Theorem 2.3. If $E_{P} W \log _{b} W=1$ but $P_{t}(W=b)<1$, then, since under the size-biasing $W>0$ a.s., one has that $\lim \sup \log \lambda_{n}(T)=\infty, P_{1, t}$-a.s. by the Chung-Fuchs null-recurrence criterion for the random walk along the $t$-path. Integrate out $t$ and use Theorems 2.2 and 2.1 (in that order) to conclude that $\lambda_{\infty}(T)=0, P$-a.s. The special case of the degeneracy $P_{t}(W=b)=1$, i.e. $P(W=b)=\frac{1}{b}=1-P(W=0)$, may be handled similarly or directly from the theory of branching processes.

In a similar manner one may obtain the Kahane-Peyrière divergence of moments criterion from Theorems 2.3, 2.2, and 2.1 (in that order). The condition for nondegeneracy and the percolation method of Theorem 2.4 may then be combined to obtain the Hausdorff dimension of the support of $\lambda_{\infty}$ in the manner first noted in [K1].

The detailed proofs for the underlying theory and applications to general classes of dependent cascades appear in the companion paper [WW3].

\section{REFERENCES}

[C] C. Cutler, The Hausdorff dimension distribution of finite measures in Euclidean space, Canad. J. Math. XXXVIII (1986).

[CK] P. Collet and F. Koukiou, Large deviations for multiplicative chaos, Comm. Math. Phys. 147 (1992), 329-342.

[CCD] J. Chayes, L. Chayes, and R. Durrett, Connectivity properties of Mandelbrot's percolation, Probab. Theory Related Fields 77 (1988), 307-324.

[DE] B. Derrida, Directed polymers in a random medium, Phys. A 163 (1990), 71-84.

[DF] L. Dubins and D. A. Freedman, Random distribution functions, Bull. Amer. Math. Soc. 69 (1963), 548-551.

[DG] F. M. Dekking and G. R. Grimmett, Superbranching processes and projections of random Cantor sets, Probab. Theory Related Fields 78 (1988), 335-355.

[DL] R. Durrett and T. M. Liggett, Fixed points of the smoothing transformation, Z. Wahr. Verw. Geb. 64 (1983), 275-301.

[DM] F. M. Dekking and R. W. J. Meester, On the structure of Mandelbrot's percolation process and other random Cantor sets, J. Stat. Phys. 58 (1990), 1109-1126.

[F] K. Falconer, The multifractal spectrum of statistically self-similar measures, preprint.

[GMW] S. Graf, D. Mauldin, and S. Williams, The exact Hausdorff dimension in random recursive constructions, Mem. Amer. Math. Soc., vol. 71, Amer. Math. Soc., Providence, RI, 1987.

[GW] V. K. Gupta and E. Waymire, A statistical analysis of mesoscale rainfall as a random cascade, J. Appl. Meteor. 32 (1993), 251-267.

[HL] R. Holley and T. M. Liggett, Generalized potlatch and smoothing processes, Z. Wahr Verw. Geb. 55 (1981), 165-195.

[HW] R. Holley and E. Waymire, Multifractal dimensions and scaling exponents for strongly bounded random cascades, Ann. Appl. Probab. 2 (1992), 819-845. 
[KP] J. P. Kahane and J. Peyrière, Sur certaines martingales de Benoit Mandelbrot, Adv. Math. 22 (1976), 131-145.

[K1] J. P. Kahane, Multiplications aléatoires et dimensions de Hausdorff, Ann. Inst. Poincaré 23 (1987), 289-296.

[K2] Positive Martingales and random measures, Chinese Ann. Math. 8 (1987), 1-12.

[K3] Random multiplications, random coverings, and multiplicative chaos, Proceedings of the Special Year in Modern Analysis (E. Berkson, N. Tenney Peck, J. Jerry Uhl, eds.), London Math. Soc. Lect. Notes, vol. 137, Cambridge Univ. Press, London, 1989, pp. 196255.

[K4] , Produits de poids aléatoires indépendants et applications, Fractal Geometry and Analysis (J. Belair and S. Dubac, eds.), Kluwer Academic Publ., The Netherlands, 1991.

[KK] J. P. Kahane and Y. Katznelson, Décomposition des mesures selon la dimension, Colloq. Math. LVII (1990), 269-279.

[L] R. Lyons, Random walks and percolation on trees, Ann. Probab. 18 (1990), 931-958.

[MS] C. Meneveau and K. R. Sreenivasan, The multifractal spectrum of the dissipation field in turbulent flows, Nuclear Phys. B (Proc. Suppl.) 2 (1987), 49-76.

[N] Ben Nasr, Mesures aléatores de Mandelbrot associées à des substitutions, C. R. Acad. Sc. Paris Sér. I Math. 304 (1987), 255-258.

[O] L. Olsen, Random geometrically graph directed self-similar multifractals, Mem. Amer. Math. Soc. (to appear).

[PW] S. Pekham and E. Waymire, On a symmetry of turbulence, Comm. Math. Phys 147 (1991), 365-370.

[P] J. Peyrière, Calculs de dimensions de Hausdorff, Duke Math. J. 44 (1977), 591-601.

[Pr] C. Preston, Gibbs states on countable sets, Cambridge Univ. Press, London, 1974.

[S] L. Shepp, Covering the line with random intervals, Z. Wahr. Verw. Geb. 23 (1972), 163170.

[Sp] F. Spitzer, Markov random fields on an infinite tree, Ann. Probab. 3 (1975), 387-398.

[TLS] Y. Tessier, S. Lovejoy, and D. Schertzer, Universal multifractals: Theory and observation for rain and clouds, J. Appl. Meteor. 32 (1993), 223-250.

[WW1] E. Waymire and S. C. Williams, Markov cascades, preprint.

[WW2] - Multiplicative cascades: Dimension spectra and dependence, Preprint presented at Colloque en l'honneur de J.-P. Kahane (June 28 - July 3, 1993), Université de Paris-Sud, Orsay, France.

[WW3] _ A cascade decomposition theory with applications to Markov and exchangeable cascades, preprint.

Mathematics Department, Oregon State University, Corvallis, Oregon 97330

E-mail address: waymire@math.orst.edu

Mathematics Department, Utah State University, Logan, Utah 84321

E-mail address: williams@sunf s.math.usu.edu 